\title{
TDM thoracique d'un des premiers cas canadiens de COVID-19 chez un homme de 28 ans
}

\author{
Navneet Singh MD PhD, Jennifer Fratesi MD
}

- Citation : CMAJ 2020 April 27;192:E455. doi : 10.1503/cmaj.200431-f; diffusion hâtive le 15 avril 2020

Voir la version anglaise de l'article ici : www.cmaj.ca/lookup/doi/10.1503/cmaj.200431

$\mathbf{U}$ n homme asthmatique de 28 ans a consulté aux services des urgences le lundi 16 mars 2020, pour malaise thoracique, toux sèche et dyspnée, malgré l'utilisation d'un inhalateur de salbutamol. Il ne présentait aucun antécédent de voyage ou de contact. Il a mentionné avoir fait de la fièvre pendant 3 à 5 jours, avoir présenté des myalgies et des arthralgies, et avoir pris de l'acétaminophène. L'examen physique a révélé des bruits respiratoires basilaires normaux et quelques sibilances éparses. Ses signes vitaux étaient stables $\left(36,8{ }^{\circ} \mathrm{C}\right.$, saturation de l'oxygène périphérique $\left[\mathrm{SpO}_{2}\right] 100 \%$, fréquence cardiaque de 85 pulsations par minute et tension artérielle à 130/79 mm Hg). Ses analyses sanguines étaient normales, sauf la protéine $C$ réactive $(32$ [normale $<11] \mathrm{mg} / \mathrm{L})$ et les lymphocytes $(0,4$ [normale $1,5-4] \times 103$ cellules $/ \mu \mathrm{L})$.

La tomodensitométrie (TDM) thoracique (figure 1) a révélé les signes typiques de la pneumonie causée par la COVID-19 (maladie à coronavirus 2019), selon le récent énoncé consensuel des experts de la Radiology Society of North America sur les signes de la COVID-19 à la TDM thoracique, notamment: opacités en verre dépoli bilatérales périphériques arrondies et zones de consolidation périphérique ${ }^{1}$. Le diagnostic radiologique différentiel incluait pneumonie bilatérale due à un agent pathogène typique ou atypique, toxicité médicamenteuse ou pneumonie en voie d'installation.

Le frottis naso-pharyngé, prélevé aux services des urgences immédiatement après la TDM, s'est par la suite révélé positif à l'égard de la COVID-19 au test RT-PCR (transcription inverse-réaction en chaîne de la polymérase, Seegene Allplex 2019-nCoV Assay), qui détecte les gènes encodant l'ARNpolymérase dépendante de l'ARN (RdRp), l'enveloppe et la nucléocapside. Le patient a été hospitalisé en médecine générale. On lui a administré de l'oseltamivir et il s'est entièrement rétabli sans devoir être admis aux soins intensifs.

Il n'est pas recommandé de procéder d'emblée à un dépistage diagnostique par TDM pour la COVID-19 en raison des coûts, de la précarité des ressources et du risque de propagation

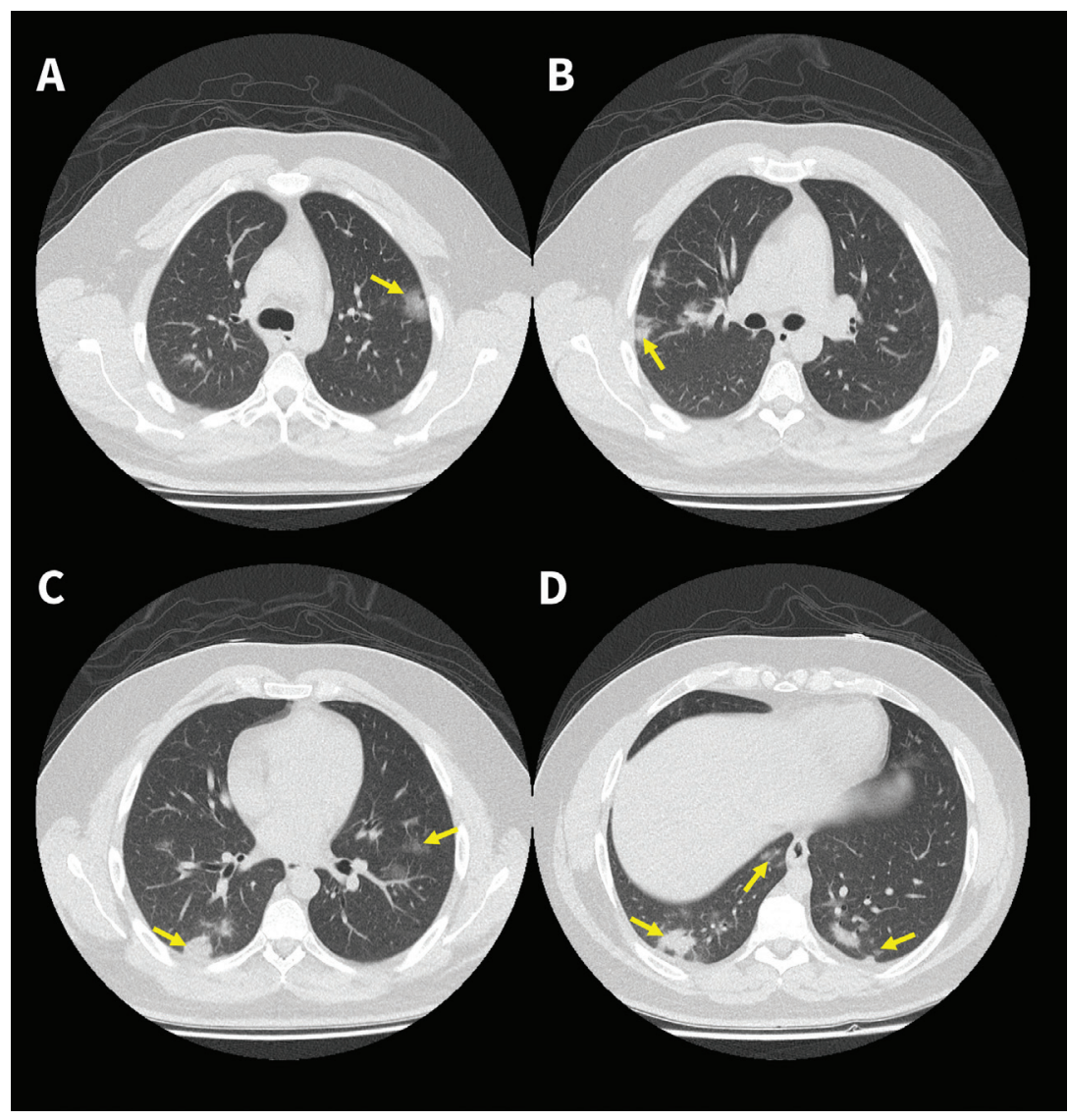

Figure 1 : Tomodensitométrie thoracique chez un homme de 28 ans qui révèle des opacités en verre dépoli multifocales bilatérales périphériques arrondies aux contours flous ( $A$ et $B$ ) avec des zones de consolidation arrondies aux lobes inférieurs (C et D).

propre à une pandémie². Mais la TDM peut être utilisée pour accélérer les soins chez des patients symptomatiques dont le frottis est négatif ou en attente de résultats, et chez ceux dont la fonction respiratoire se détériore ou qui développent des complications telles que l'empyème ou un syndrome de détresse respiratoire aigu. Selon une récente étude sur 601 patients, la TDM thoracique serait sensible à $97 \%$, mais spécifique à $25 \%$ seulement, comparativement au test RT-PCR ${ }^{3}$, qui est sensible et spécifique $(95 \%-97 \%)^{4}$. Ce cas canadien met en lumière les caractéristiques de la COVID-19 à l'imagerie, ce qui concorde avec l'expérience internationale ${ }^{5}$. 


\section{Références}

1. Simpson S, Kay FU, Abbara S, et al. Chest CT findings related to COVID-19. Endorsed by the Society of Thoracic Radiology, the American College of Radiology, and RSNA. Radiology: Cardiothoracic Imaging 2020 le 25 mars. doi. org/10.1148/ryct.2020200152.

2. ACR Recommendations for the use of chest radiography and computed tomography (CT) for suspected COVID-19 infection. Reston (VA): American College of Radiology; mise à jour 2020 le 22 mars. Accessible ici : www.acr.org/Advocacy-and -Economics/ACR-Position-Statements/Recommendations-for-Chest-Radiography -and-CT-for-Suspected-COVID19-Infection (consulté le 17 mars 2020).

3. Ai T, Yang Z, Hou H, et al. Correlation of chest CT and RT-PCR testing in Coronavirus disease 2019 (COVID-19) in China: a report of 1014 cases. Radiology 2020 le 26 février:200642. doi: 10.1148/radiol.2020200642.

4. Mossa-Basha M, Meltzer CC, Kim DC, et al. Radiology department preparedness for COVID-19: Radiology Scientific Expert Panel. Radiology 2020 le 16 mars:200988. doi: 10.1148/radiol.2020200988.

5. Shi H, Han X, Jiang N, et al. Radiological findings from 81 patients with COVID-19 pneumonia in Wuhan, China: a descriptive study. Lancet Infect Dis 2020 le 24 février. doi.org/10.1016/ S1473-3099(20)30086-4.

Intérêts concurrents : Aucun déclaré.

Cet article a été révisé par des pairs.

Les auteurs ont obtenu le consentement du patient.

Affiliations : Département d'imagerie médicale, Faculté de médecine, Université de Toronto; Hôpital général de Toronto, Département mixte d'imagerie médicale, Réseau universitaire de santé, Toronto, Ont.

Adresse de correspondance : Navneet Singh, navneet.singh@ utoronto.ca 